\title{
Correlation between peripheral blood cell transcriptomic profile and clinical parameters of chronic mitral valve disease in Dachshunds
}

\author{
M. Garncarz ${ }^{1}$, M. Hulanicka², H. Maciejewski ${ }^{3}$, \\ M. Parzeniecka-Jaworska1, M. Jank ${ }^{4}$ \\ ${ }^{1}$ Department of Veterinary Diagnostics and Pathology, Faculty of Veterinary Medicine, \\ Warsaw University of Life Sciences, Warsaw, Nowoursynowska159, 02-766 Warsaw, Poland \\ ${ }^{2}$ Department of Physiological Sciences, Faculty of Veterinary Medicine, \\ Warsaw University of Life Sciences, Warsaw, Poland \\ ${ }^{3}$ Institute of Computer Engineering, Control and Robotics (I-6), \\ Wroclaw University of Technology, Wroclaw, Poland \\ ${ }^{4}$ Veterinary Institute, Faculty of Veterinary Medicine and Animal Sciences, \\ Poznań University of Life Sciences, Poznań, Poland
}

\begin{abstract}
Studies identifying specific pathologically expressed genes have been performed on diseased myocardial tissue samples, however less invasive studies on gene expression of peripheral blood mononucleated cells give promising results. This study assessed transcriptomic data that may be used to evaluate Dachshunds with chronic mitral valve disease. Dachshunds with different stages of heart disease were compared to a control, healthy group. Microarray data analysis revealed clusters of patients with similar expression profiles. The clusters were compared to the clinical classification scheme. Unsupervised classification of the studied groups showed three clusters. Clinical and laboratory parameters of patients from the cluster 1 were in accordance with those found in patients without heart disease. Data obtained from patients from the cluster 3 were typical of advanced heart failure patients. Comparison of the cluster 1 and 3 groups revealed 1133 differentially expressed probes, 7 significantly regulated process pathways and 2 significantly regulated Ariadne Metabolic Pathways. This study may serve as a guideline for directing future research on gene expression in chronic mitral valve disease.
\end{abstract}

Key words: dogs, heart failure, transcriptomic profile, clinical, blood cells 


\section{Introduction}

Chronic mitral valve disease (CMVD) appears to have a high prevalence in some breeds, the Dachshund being one (Garncarz et al. 2013). Possible factors implicated in the disease include genetics (Abbott 2008). Olsen et al. (1999) have postulated that a polygenic character of inheritance may be responsible for the disease in Dachshunds, while Madsen et al. (2011) have implicated two loci in CMVD in the Cavalier King Charles Spaniel (CKCS), another breed affected by the disease. These publications underlie the role genetics play in this acquired disease (Lewis et al. 2011). French et al. (2012) have shown that the disease is not a result of a single gene effect in the CKCS, emphasizing the necessity to find specific mutations when making decisions related to breeding dogs. Stern et al. (2015) have shown that disease severity may also be associated with genetic factors in Whippets.

To elucidate the role of genetics in development of CMVD studies have been undertaken to identify specific genes which, when pathologically expressed, lead to heart lesions or dysfunction. Most of these studies have been performed on tissue samples taken from the diseased hearts (Oyama et al. 2005, Disatian et al. 2008, Zheng et al. 2009, Aupperle et al. 2010, Obayashi et al. 2011). The major drawback of these studies is the difficult in vivo acquisition of diagnostic material via i.e. biopsy. Studies on gene expression of peripheral blood nucleated cells seem a feasible alternative. Studies have shown changes in the expression of $\mathrm{Na}+-\mathrm{Ca} 2+$ exchanger (NCX-1) responsible for removing calcium from cells (Moon et al. 2008, Nam et al. 2010) and phospholamban (PLN), which regulates the $\mathrm{Ca} 2+$ pump in cardiac muscles (Mishra et al. 2005, Lee et al. 2009). Hulanicka et al. (2014) showed significant differences in the expression of genes from isolated RNA samples from peripheral blood nuclear cells (PBNC) between dogs with different stages of CMVD postulating $\alpha$-SMA as a potential biomarker of heart failure.

The dataset of transcriptomic profiles (Gene Expression Omnibus data repository under the number GSE48319) of Dachshund dogs with heart failure secondary to CMVD was analysed and correlated with all clinical and laboratory data obtained from these animals. The main aim of this study was to verify whether transcriptomic profiles of peripheral blood nuclear cells of Dachshunds with CMVD are correlated with any clinical and laboratory data routinely evaluated. For this purpose we wanted to (i) identify groups (clusters) of patients with similar expression profiles and (ii) identify clinical variables, which are significantly associated with these clusters and (iii) compare the clusters in terms of differentially expressed genes.

\section{Materials and Methods}

This study complies with national and institutional guidelines on the use of animals in clinical research according to the Polish legal act from January 21st, 2005 concerning experiments performed on client owned animals. An informed consent was obtained from dog owners and a high standard of care was adhered to. A prospective study and data analysis were carried out on dogs submitted to the Faculty of Veterinary Medicine Cardiology Service, Warsaw School of Agriculture. Dachshunds with recognized heart disease were classified according the ACVIM (American College of Veterinary Internal Medicine) classification scheme (Atkins et al. 2009). Initially 52 Dachshunds were examined, however 29 dogs were eliminated from further studies due to recognized and significant comorbidities. The remaining 23 dogs were grouped as follows: class B - 6 dogs, class C -6 dogs, class D - 6 dogs, class A (control group) - 5 healthy dogs. Study population characteristics are shown in Table 1.

Clinical and laboratory examinations were performed according to accepted methods (Abbott 2008). A transthoracic echocardiographic examination (Aloka 4000 with $2.5-7 \mathrm{mHz}$ sector transducers) was performed at rest without pharmacological restraint according to standard procedures in right and left lateral recumbency, in some cases in the standing position (Abbott 2008) and allowed for diagnosis and staging of mitral valve disease. A six lead ECG was performed with BTL-08 MD machine in the dogs in right lateral recumbency position.

For laboratory testing blood samples were collected via venipuncture from the cephalic or jugular veins. Basic morphologic and biochemical testing was performed in the Laboratory of The Small Animal Clinic, including a complete blood count, serum sodium, potassium, urea, creatinine, total protein, aspartate transaminase (ASpAT), alanine aminotransferase (AlAT), alkaline phosphatise (AP) and glucose. For RNA testing blood samples were collected in Rneasy Protect Animal Blood Tubes (Qiagen, USA). Total RNA from peripheral blood nuclear cells was isolated with Rneasy Protect Animal Blood Kit (Qiagen, USA). Isolated RNA samples were dissolved in $30 \mu \mathrm{l}$ of REB Buffer from the test kit. RNA quantity was measured spectrophotometrically using a NanoDrop (NanoDrop Technologies, USA). Final RNA quality and integrity was analysed with a BioAnalyzer (Agilent, USA). To ensure optimal data quality, only RNA samples with RIN number $\geq 7.5$ were included in the analysis.

Analysis of gene-expression profiles was performed using Canine (V2) Gene Expression Microar- 
Table 1. Clinical characteristics of the study population in the control, ACVIM B, C, and D groups of Dachshunds.

\begin{tabular}{|c|c|c|c|c|}
\hline ACVIM stage & $\begin{array}{l}\text { A unaffected } \\
(\text { mean } \pm S D)\end{array}$ & $\begin{array}{c}\mathrm{B} \\
(\text { mean } \pm \mathrm{SD})\end{array}$ & $\begin{array}{c}\mathrm{C} \\
(\text { mean } \pm \mathrm{SD})\end{array}$ & $\begin{array}{c}\mathrm{D} \\
(\text { mean } \pm \mathrm{SD})\end{array}$ \\
\hline Number of dogs & 5 & 6 & 6 & 6 \\
\hline Age range (months) & $59.60 \pm 9.74^{\mathrm{a}}$ & $116.7 \pm 34.39^{\mathrm{b}}$ & $162.50 \pm 17.81^{\mathrm{c}}$ & $153.20 \pm 13.24^{\mathrm{c}}$ \\
\hline \multirow{2}{*}{ Sex $\frac{\text { Males }}{\text { Females }}$} & 1 & 2 & 4 & 5 \\
\hline & 4 & 4 & 2 & 1 \\
\hline Body weight (kg) & $9.10 \pm 2.79$ & $10.00 \pm 1.64$ & $10.45 \pm 2.82$ & $11.43 \pm 3.84$ \\
\hline Heart rate & $117.80 \pm 31.87$ & $116.30 \pm 20.02$ & $146.50 \pm 22.02$ & $158.80 \pm 34.83$ \\
\hline $\mathrm{EF}$ & $69.40 \pm 11.13^{\mathrm{a}}$ & $73.33 \pm 7.71^{\mathrm{ab}}$ & $81.17 \pm 7.08^{\mathrm{ab}}$ & $83.33 \pm 4.84^{\mathrm{b}}$ \\
\hline LA/Ao & $1.32 \pm 0.125^{\mathrm{a}}$ & $1.36 \pm 0.21^{\mathrm{a}}$ & $2.16 \pm 0.43^{\mathrm{b}}$ & $2.22 \pm 0.35^{\mathrm{b}}$ \\
\hline $\mathrm{LA} \mathrm{cm}$ & $1.83 \pm 0.187^{\mathrm{a}}$ & $2.17 \pm 0.30^{\mathrm{a}}$ & $3.37 \pm 0.65^{b}$ & $3.47 \pm 0.46^{\mathrm{b}}$ \\
\hline LVDd cm & $2.77 \pm 0.52^{\mathrm{a}}$ & $3.10 \pm 0.39^{\mathrm{ac}}$ & $3.78 \pm 0.65^{\mathrm{bc}}$ & $4.57 \pm 0.50^{\mathrm{b}}$ \\
\hline $\mathrm{MVR} \mathrm{m/s}$ & $0.0 \pm 0.0^{\mathrm{a}}$ & $5.17 \pm 1.05^{\mathrm{b}}$ & $5.80 \pm 0.96^{\mathrm{b}}$ & $5.08 \pm 0.41^{\mathrm{b}}$ \\
\hline $\mathrm{RVDd} \mathrm{cm}$ & $0.74 \pm 0.16$ & $0.66 \pm 0.15$ & $0.68 \pm 0.18$ & $0.68 \pm 0.25$ \\
\hline $\mathrm{TVR} \mathrm{m/s}$ & $0.21 \pm 0.48$ & $1.46 \pm 1.64$ & $2.16 \pm 1.92$ & $2.30 \pm 1.86$ \\
\hline
\end{tabular}

Data are shown as mean \pm standard deviation (SD). Values in each row marked with different superscripts (a, b, c) are statistically different. ACVIM - American College of Veterinary Internal Medicine.

HR - heart rate, EF - ejection fraction, LA/Ao - left atrial to aortic root ration, LA - left atrium, LVDd - left ventricular diameter in diastole, MVR - mitral valve regurgitation, RVDd - right ventricular diameter diastole, TVR - tricuspid valve regurgitation

ray, 4x44K (Agilent Technologies, USA). Each slide contained 4 microarrays representing about 45000 canine predicted mRNAs. The Quick Amp Labelling Kit (Agilent, USA) was used to amplify and label target RNA to generate complementary RNA (cRNA) for oligo microarrays used in gene expression profiling. A common reference pool of equal amounts of RNA from 10 healthy Dachshund dogs was used. On each two-colour microarrays, $825 \mathrm{ng}$ of cRNA (labelled Cy3) and 825 ng of cRNA (labelled Cy5) was hybridized from the pool. Microarray hybridization was performed with Gene Expression Hybridization Kit (Agilent Technologies, USA) according to the manufacturer's protocols. RNA Spike In Kit (Agilent Technologies, USA) was used as an internal control. Acquisition and analysis of hybridization intensities were performed using Agilent DNA microarray scanner.

Data were extracted and background subtracted using standard procedures contained in the Agilent Feature Extraction (FE) Software version 10.7.3.1. FE performs a Lowess normalization. Statistical analysis was performed using Gene Spring 12 software (Agilent, USA). The samples underwent quality control and the results showed that each sample had a similar QC metric profile. Next filtering probe sets by flags were used to remove poor quality probes (absent flags). The statistical significance of the differences was evaluated using a one-way ANOVA and Tukey's HSD Post-hoc test $(\mathrm{p}<0.05)$. A multiple test- ing correction was performed using Benjamini and Hochberg False Discovery Rate (FDR) with significant differences indicated by the condition FDR-corrected $\mathrm{p}$-value $<5 \%$. Microarray data were deposited at the Gene Expression Omnibus data repository under number GSE48319 and followed MIAME requirements.

Unsupervised analysis was performed using hierarchical clustering with Ward's minimum variance method. We tested which of the clinical variables were significantly associated with cluster ID using the Chi-square test and Fisher exact (for testing association of the cluster ID with discrete clinical variables), and the nonparametric Wilcoxon and Kruskall-Vallis tests (for association of cluster ID with continuous clinical variables).

Finally, we analyzed differentially expressed genes between the clusters identified by the unsupervised analysis using linear methods for microarrays (the limma R/Bioconductor package) (Smyth 2004, Ritchie 2015). In this procedure, we first performed unspecific filtering of the expression data to remove probes with no expression (technically, probes with mean expression over all samples below 100 were considered probes with no expression); then we calculated signal as median-centered $\log 2$ ratio of $\mathrm{R}$ vs $\mathrm{G}$ channel, and used these signal values in the limma procedure for differential expression analysis, based on the lmFit function, with the target taken as the 


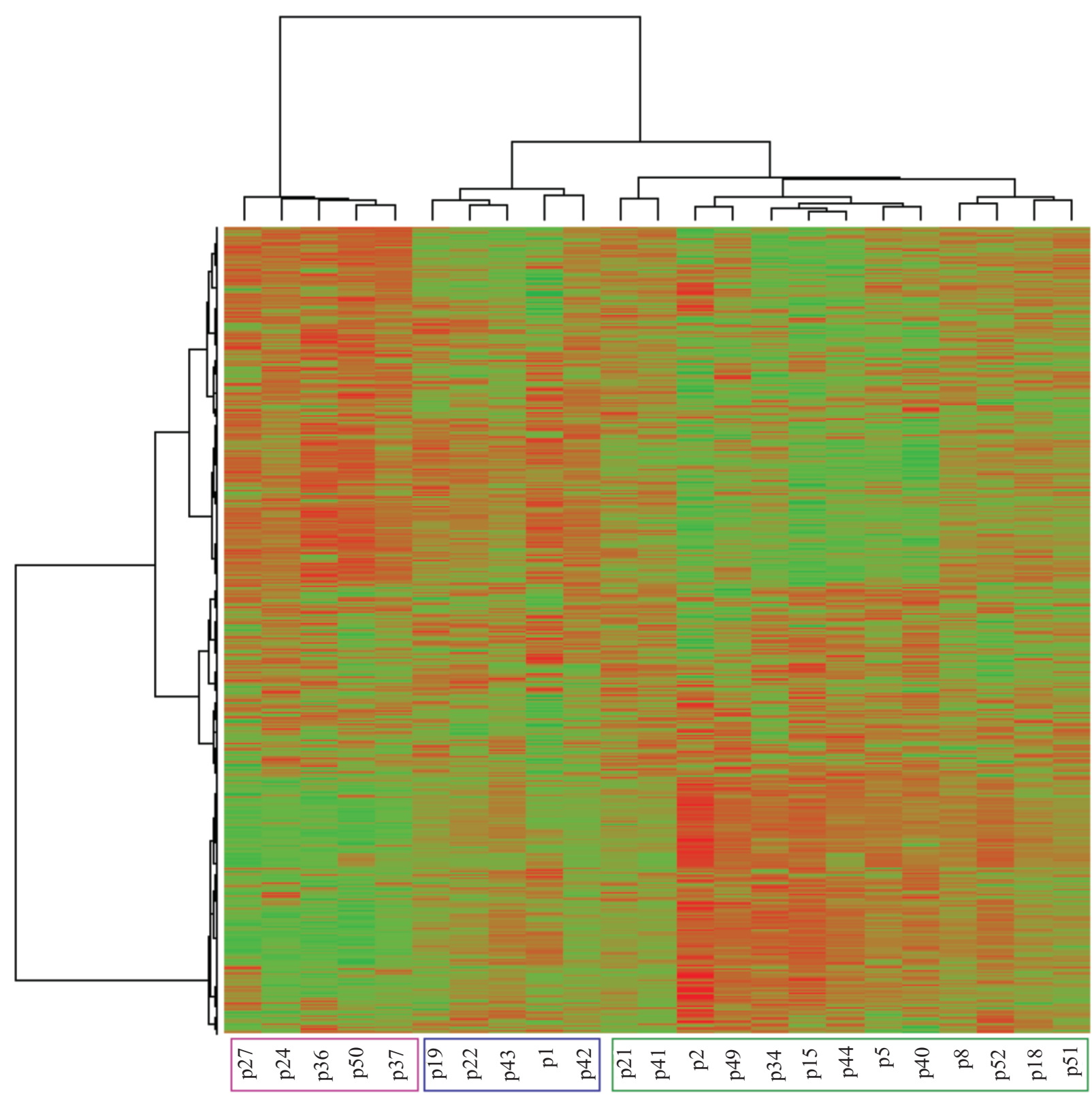

Fig. 1. Hierarchical clustering of gene expression data with rows corresponding to genes and columns to samples (patients). Unsupervised classification (Ward method) of 20000 most variable genes. Three clusters are presented (cluster 1-purple box, 2- green box, 3-blue box). Each gene is represented by a colour bar. Up-regulated genes are denoted in green, down-regulated in red.

cluster ID (Smyth 2004). Statistical tests for association between cluster ID and clinical data were performed with SAS/STAT 12.3 software; all remaining analyses were performed using the R/Bioconductor system (Huber 2015).

Pathway analysis of the obtained set of differentially expressed genes was performed using Ariadne's Pathway Studio software (Ariadne Genomics, USA), which interprets gene expression and other high throughput data; builds, expands and analyzes pathways; finds relationships among genes, proteins, cell processes and diseases; and draws pathway diagrams. The software gives information on the different types of pathways, namely cell process pathways, metabolic pathways, receptor pathways, cell signalling pathways.

\section{Results}

The key cardiologic data are consistent with the intensity of heart disease and the classification to specific ACVIM group (Table 1).

The results of the unsupervised classification are presented in Fig. 1. Cluster analysis revealed three groups of patients with distinct gene expression profiles: cluster 1 with patients p24, p27, p36, p37 and 
Table 2. Clinical and laboratory parameters analysed for correlation with peripheral blood mononuclear cell transcriptomic cluster profiles 1, 2 and 3 of the Dachshunds studied.

\begin{tabular}{lccc}
\hline \multicolumn{1}{c}{ Parameter } & Cluster 1 & Cluster 2 & Cluster 3 \\
\hline Patients no. & $24,27,36,37,50$ & $2,5,8,15,18,21,34,40,41,44,49,51,52$ & $1,19,22,42,43$ \\
\hline AVCIM classification & ACVIM A $(\mathrm{n}=2) ;$ & ACVIM A $(\mathrm{n}=3)$ & ACVIM B (n=2) \\
& ACVIM B $(\mathrm{n}=3)$ & ACVIM B $(\mathrm{n}=1)$ & ACVIM D (n=3) \\
& & ACVIM C (n=6) & \\
\hline
\end{tabular}

\begin{tabular}{|c|c|c|c|}
\hline Respiratory arrhythmia & $4 / 5$ & $5 / 13$ & $0 / 5$ \\
\hline Tachycardia $>140 / \mathrm{min}$ & $1 / 5$ & $7 / 13$ & $5 / 5$ \\
\hline Average HR & $116 \pm 23^{\mathrm{a}}$ & $143 \pm 33^{\mathrm{ab}}$ & $170 \pm 24^{b}$ \\
\hline Age (months) & $97.2 \pm 49.1$ & $134.5 \pm 46.7$ & $131.6 \pm 25.7$ \\
\hline Murmur & $1.8 \pm 1.8$ & $3.4 \pm 2.5$ & $3.6 \pm 1.1$ \\
\hline LA/Ao & $1.22 \pm 0.12^{\mathrm{a}}$ & $1.89 \pm 0.46^{\mathrm{b}}$ & $2.04 \pm 0.55^{\mathrm{b}}$ \\
\hline LVWs cm & $0.92 \pm 0.08^{\mathrm{a}}$ & $1.22 \pm 0.19^{\mathrm{b}}$ & $1.15 \pm 0.09^{\mathrm{ab}}$ \\
\hline FS \% & $34.2 \pm 4.9^{\mathrm{a}}$ & $48.3 \pm 7.1^{b}$ & $48.2 \pm 2.0^{\mathrm{b}}$ \\
\hline $\mathrm{EF} \%$ & $64.6 \pm 6.4^{\mathrm{a}}$ & $80.8 \pm 7.1^{\mathrm{b}}$ & $80.0 \pm 4.5^{\mathrm{b}}$ \\
\hline EPSS cm & $0.33 \pm 0.16$ & $0.18 \pm 0.09$ & $0.25 \pm 0.11$ \\
\hline $\mathrm{N}$ segm. $\%$ & $55.2 \pm 6.1^{\mathrm{a}}$ & $69.7 \pm 9.4^{\mathrm{b}}$ & $73.0 \pm 12.0^{\mathrm{b}}$ \\
\hline AlAT U/1 & $30.0 \pm 9.9$ & $78.1 \pm 75.7$ & $73.7 \pm 20.0$ \\
\hline $\mathrm{K} \mathrm{mmol} / \mathrm{l}$ & $4.85 \pm 0.52$ & $4.32 \pm 1.05$ & $4.74 \pm 0.30$ \\
\hline P wave amp. & $0.29 \pm 0.07$ & $0.37 \pm 0.11$ & $0.31 \pm .06$ \\
\hline $\mathrm{P}$ wave duration $\mathrm{s}$ & $0.036 \pm 0.005^{\mathrm{a}}$ & $0.047 \pm 0.008^{b}$ & $0.050 \pm 0.007^{\mathrm{b}}$ \\
\hline $\mathrm{R}$ wave amp. MV & $1.29 \pm 0.82$ & $2.07 \pm 1.01$ & $2.41 \pm 1.30$ \\
\hline QRS duration $\mathrm{s}$ & $0.054 \pm 0.008$ & $0.054 \pm 0.017$ & $0.064 \pm 0.005$ \\
\hline
\end{tabular}

Data are shown as mean \pm standard deviation (SD). Values in each row marked with different superscript (a and b) are significantly different $(\mathrm{p}<0.05)$. ACVIM-American College of Veterinary Internal Medicine.

HR - heart rate, LA/Ao - left atrial to aortic root ration, LVWs - left ventricular wall in systole, FS - fractional shortening, EF - ejection fraction, EPSS - end point to septal separation, N segm. - segmented neutrophils, AlAT - alanine aminotransferase, $\mathrm{K}$ - potassium, amp. - amplitude.

p50; cluster 2 with patients p2, p5, p8, p15, p18, p21, p34, p40, p41, p44, p49, p51 and p52, and cluster 3 with patients $\mathrm{p} 1, \mathrm{p} 19, \mathrm{p} 22, \mathrm{p} 43, \mathrm{p} 42$.

The groups revealed in the cluster analysis were then correlated with clinical data for symptoms, laboratory, ECG and echocardiographic findings (Table 2). Based on these data we concluded that patients belonging to the cluster 1 group might be considered as "healthy" ones, whereas patients belonging to the cluster 3 group could be considered ones with more advanced heart disease. Patients from the cluster 2 have a more scattered transcriptomic cluster profile in between that of cluster 1 and 3 .

A comparison of gene expressions between the two clearly different cluster groups -1 and 3 revealed 1133 differentially expressed probes (FDR-adjusted $\mathrm{p}<0.01$ ) (data not shown). The data were re-clustered in the space of these 1133 differentially expressed genes as shown in Fig. 2. Ontology analysis using the Ariadne software revealed 7 significantly regulated process pathways and 2 significantly regulated Ariadne Metabolic Pathways (Table 3).

\section{Discussion}

The search for the new biomarkers of specific diseases has recently become a very important direction in science and research. Wide access to many large-scale scientific tools and methods often results in thousands of data obtained in just one experiment. This frequently leads to many new hypotheses rather than simple and clear answers.

In our previous study we were able to show that the transcriptomic profile of peripheral blood mononuclear cells (PBMC) in humans after acute heart failure could be correlated with their kidney function and increased plasma creatinine levels (Szmit et al. 2012). In the present study, we wanted to find out whether the classification of patients based on tran- 


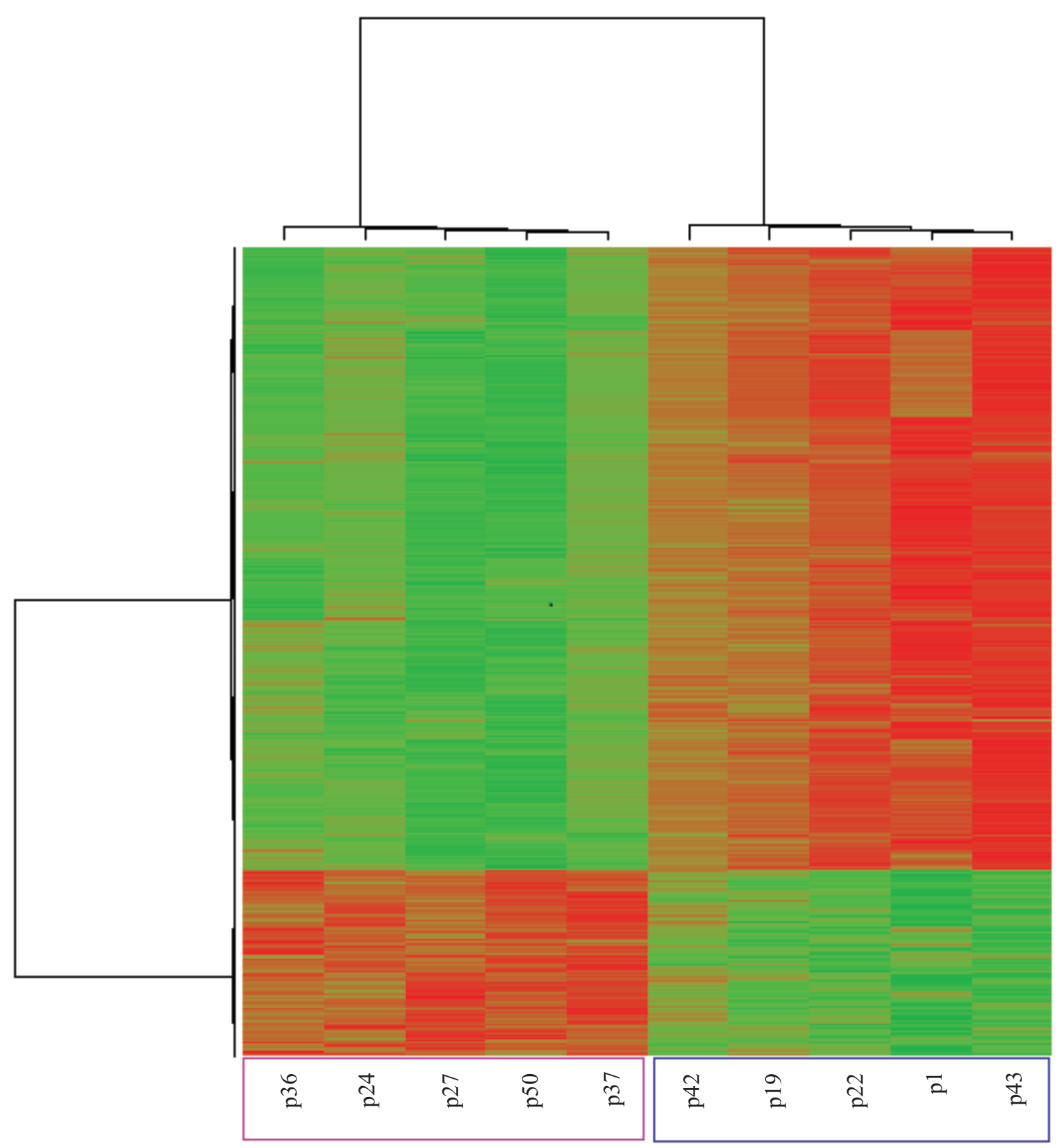

Fig. 2. Hierarchical clustering between cluster 1 (purple box) and 3 (blue box) in the space of $n=1133$ differentially expressed genes.

scriptomic profiling results of their PBMC could be useful for the clinical staging of CMVD. Although the disease is well known from the clinical point of view, its origin is still a matter of debate so the methodology used in the present study was also aimed at searching for new potential biomarkers of this disease or trying to elucidate disease pathogenesis. In fact the analysis revealed the presence of three clusters. The cluster 1 group represented dogs from ACVIM A (healthy) and ACVIM B (subclinical heart failure), logically grouping dogs with no clinical symptoms. The cluster 2 group included the most diverse patients. This in fact is a reflection of the greatest challenge for veterinary practitioners - staging patients in their disease severity, and also underlines the need for further studies that might finally show us one or several precise markers of patient disease stage.

The cluster 3 group consisted of 2 ACVIM B patients and 3 ACVIM D patients - the discrepancy seems to be significant. However the clear difference seen between transcriptomic profiles of the cluster 1 and 3 groups cannot be denied. The $n=1133$ differentially regulated genes (Fig. 2) between these two clusters seem to be important and we believe deemed 
Table 3. Differentially regulated Ariadne pathways in peripheral blood nuclear cells of two groups of Dachshunds with heart failure severity based on results of cluster hierarchical analysis of transcriptomic profiles.

\begin{tabular}{|c|c|c|c|}
\hline Name & Data Source & Significantly regulated genes & p-value \\
\hline $\begin{array}{l}\text { Tight Junction Assembly } \\
\text { (JAMs) }\end{array}$ & \multirow{7}{*}{$\begin{array}{l}\text { Ariadne Cell } \\
\text { Process Pathways }\end{array}$} & $\begin{array}{c}\text { ESAM; MYH7; CLDN6; CLDN4; MYH7B; } \\
\text { TJAP1; PARD3B }\end{array}$ & 0.0157 \\
\hline $\begin{array}{l}\text { Double Strand DNA Homologous } \\
\text { Repair }\end{array}$ & & $\begin{array}{l}\text { PARP1; REV1; FANCG; DDB1; POLH; } \\
\text { RECQL; RFC5; RECQL5; RBBP8 }\end{array}$ & 0.0198 \\
\hline $\begin{array}{l}\text { Single-Strand Mismatch DNA } \\
\text { Repair }\end{array}$ & & $\begin{array}{l}\text { PARP1; REV1; FANCG; DDB1; RECQL; } \\
\text { RFC5; RECQL5 }\end{array}$ & 0.0201 \\
\hline Adherens Junction Assembly (Nectin) & & $\begin{array}{c}\text { ESAM; CLDN6; CLDN4; WASF1; TJAP1; } \\
\text { PARD3B }\end{array}$ & 0.0270 \\
\hline Tight Junction Assembly (Claudins) & & $\begin{array}{c}\text { ESAM; MYH7; CLDN6; CLDN4; MYH7B; } \\
\text { TJAP1; PARD3B }\end{array}$ & 0.0314 \\
\hline Histone Phosphorylation & & $\begin{array}{l}\text { PARP1; AURKB; REV1; FANCG; STAG1; } \\
\text { DDB1; CSNK2A1; RECQL; CBX5; RECQL5 }\end{array}$ & 0.0464 \\
\hline $\begin{array}{l}\text { Single-Strand Base Excision DNA } \\
\text { Repair }\end{array}$ & & $\begin{array}{l}\text { PARP1; REV1; FANCG; DDB1; RECQL; } \\
\text { RECQL5 }\end{array}$ & 0.0493 \\
\hline $\begin{array}{l}\text { Inositol phosphate } \\
\text { metabolism }\end{array}$ & \multirow{2}{*}{$\begin{array}{l}\text { Ariadne Metabolic } \\
\text { Pathways }\end{array}$} & $\begin{array}{l}\text { INPPL1; PLCL1; PIP4K2A; GDPD2; ITPKA; } \\
\text { PIK3R4; NUDT4 }\end{array}$ & 0.0087 \\
\hline Vitamin $\mathrm{K}$ metabolism & & BGLAP; F9; GGCX & 0.0358 \\
\hline
\end{tabular}

further analysis. Investigating the relation of the transcriptomic profile with 75 different clinical, blood morphological, biochemical and echocardiographic parameters showed some significant differences some of which were consistent with advanced stages of heart failure in the course of CMVD in cluster 3 patients. For example the average heart rate, left-atrial to aortic ratio, fractional shortening and ejection fraction are understandably increased in the cluster 3, and even the cluster 2 albeit to different degrees, as these are typical signs of more advanced CMVD (Table 2). Both dogs from the ACVIM B group found in the cluster 3 group had a sinus rhythm over 120/min without a respiratory arrhythmia, however neither had a loud heart murmur and both had only mildly enlarged left atrial size. It must be underlined that the ACVIM B group consists of dogs without clinical signs but with chamber enlargement and it might very well be that these were dogs that were in a stage just prior to the appearance of clinical signs. We decided to check this and interestingly found that dog 19 was classified as ACVIM $\mathrm{C}$ three months after the initial examination, however dog 1 was classified as ACVIM $\mathrm{C}$ after 1.5 years. In both cases, however, significant tricuspid regurgitation was noted 3 months (dog 19) and 4 months (dog 1) after initial examination. Definitely further studies would be needed on a much larger scale to eliminate such discrepancies. Also it might be beneficial to look at other parameters to confirm that the same animals would give us similar transcriptomic cluster profiles, i.e. murmur intensity, mitral regurgitation indices, severity of mitral valve lesions. Furthermore, if this proves true, recognizing a cluster 3 type of transcriptome might be used for studies on prognostic markers for imminent heart failure.

Similarly cluster 1 type of transcriptomic profile grouped dogs that had small left atria, sinus arrhythmia and a normal heart rate. This would be consistent with mild CMVD, including dogs in the ACVIM B group (ACVIM B1 - valvular changes without chamber enlargement).

The really surprising result was that although cluster 3 patients could be characterised as ill ones and cluster 1 could be characterised as healthy ones there was no statistical difference between age of these two groups (cluster $3=131.6 \pm 11.5$ months vs. cluster $1=97.2 \pm 21.9$ months; $p=0.222$ ). The significant age difference between the AVCIM Stage groups (see Table 1) was not unexpected because the advanced staging of heart disease in dogs is clearly related with patient ageing. So logically the "ill" group should be significantly older than the "healthy" one. Of course, the question remains whether the age differences could influence transcriptomic profiles. Up to date there have been no published data about such a phenomenon in dogs. On the other hand, our hierarchical clustering gave us clusters of patients, which did not differ significantly in age and this also suggests that the differences in transcriptomic profiles of identified clusters were not related with patient age but with patient health status. However, we cannot fully eliminate the influence of age on the genomic profile of the groups and further studies would need to be performed to specifically prove this influence in dogs. 
This study shows new directions in genomics of CMVD, especially for investigating and elucidating transcriptomic changes observed in the cluster 3 group. The precise differences in the cluster groups signify a clear grouping of the animals. It may be that these groups do not signify the stage of disease but the reason some dogs progress into advanced stages of CMVD, while others remain in the subclinical phase. The list of significantly regulated genes between the two clusters seems quite long, however the list of significantly regulated pathways is short and unspecific (Table 3).

Of course this study is of typical academic value and some important questions must be asked. First, are the peripheral blood nuclear cells a reliable material for investigating heart disease in dogs? Although some doubts still exist, the last decade has shown a huge development in the field of "transcriptional bloodomics" in human (Seiler et al. 2004, Mohr and Liew 2007, Szmit et al. 2012) and veterinary medicine (Farabaugh et al. 2004, Hulanicka et al. 2014). So the question whether the results observed are solely reflecting the clinical status of the patients, are related with etiopathogenesis of heart disease, or are a result of the disease process is still open. However, the answer to the question whether peripheral blood nuclear cells can serve as a reliable material for investigating heart disease in dogs seems to be, yes.

\section{Conclusions}

Our study showed several important aspects of transcriptomic studies. Firstly, the expression of genes in peripheral blood nuclear cells could be used for studies related to different stages of heart disease in Dachshunds with CMVD. Secondly, our results can serve as a guide in directing further studies with emphasis placed on the pathways (Table 3 ) that were regulated with significant differences between dogs with no clinical signs (cluster 1) and with clinical signs (cluster 3). The drawbacks of the study include a low number of dogs and high cost of mRNA studies.

\section{Acknowledgements}

The preparation of this paper was supported by grant No. N N308 607538 received from the Polish Ministry of Science.

\section{References}

Abbott JA (2008) Acquired Valvular Disease. In: Tilley LP, Smith FWK Jr, Oyama MA, Sleeper MM (eds) Manual of Canine and Feline Cardiology. 4th ed., Saunders Elsevier, St. Louis, pp 110-138.

Atkins C, Bonagura J, Ettinger S, Fox P, Gordon S, Haggstrom J, Hamlin R, Keene B, Luis-Fuentes V, Stepien R. (2009) Guidelines for the Diagnosis and Treatment of Canine Chronic Valvular Heart Disease. J Vet Intern Med 23: 1142-1150.

Aupperle H, März I, Thielebein J, Dinges G, Schoon $\mathrm{H}$ (2010) Histomorphological findings and expression of matrix metalloproteinases and their tissue specific inhibitors (TIMPs) in normal tricuspid valves and in chronic tricuspid valvular disease in dogs. Vet J 183: 176-183.

Disatian S, Ehrhart EJ 3rd, Zimmerman S, Orton EC (2008) Interstitial cells from dogs with naturally occurring myxomatous mitral valve disease undergo phenotype transformation. J Heart Valve Dis 17: 402-411.

Farabaugh AE, Freeman KM, Rush JE, George KL (2004) Lymphocyte Subpopulations and Hematologic Variables in Dogs with Congestive Heart Failure. J Vet Intern Med 18: 505-509.

French AT, Ogden R, Eland C, Hemani G, Pong-Wong R, Corcoran B, Summers KM (2012) Genome-wide analysis of mitral valve disease in Cavalier King Charles Spaniels. Vet J 193: 283-286.

Garncarz M, Parzeniecka-Jaworska M, Jank M, Łój M (2013) A retrospective study of clinical signs and epidemiology of chronic valve disease in a group of 207 Dachshund dogs in Poland. Acta Vet Scand 55: 52.

Huber W, Carey VJ, Gentleman R, Anders S, Carlson M, Carvalho BS, Bravo HC, Davis S, Gatto L, Girke T, Gottardo R, Hahne F, Hansen KD, Iriyarrz RA, Lawrence M, Love MI, MacDonald J, Obenchain V, Oles AK, Pages H, Rezes A, Shannon P, Smzth GK, Tenenbaum D, Waldron L, Morgan M (2015) Orchestrating high-throughput genomic analysis with Bioconductor. Nat Methods 12: 115-121.

Hulanicka M, Garncarz M, Przeniecka-Jaworska M, Jank M (2014) The transcriptomic profile of peripheral blood nuclear cells in dogs with heart failure. BMC Genomics 15: 509.

Lee JS, Pak SI, Hyun C (2009) Calcium reuptake related genes as a cardiac biomarker in dogs with chronic mitral valvular insufficiency. J Vet Intern Med 23: 832-839.

Lewis T, Swift S, Woolliams JA, Blott S (2011) Heritability of premature mitral valve disease in Cavalier King Charles Spaniels. Vet J 188: 73-76.

Madsen MB, Olsen LH, Haggström J, Hoglund K, Ljungvall I, Falk T, Wess G, Stephenson H, Dukes-McEwan J, Chetboul V, Gouni V, Proschowsky HF, Cirera S, Karlskov-Mortensen P, Fredholm M (2011) Identification of 2 loci associated with development of myxomatous mitral valve disease in Cavalier King Charles Spaniels. J Hered (Suppl 1): S62-67.

Mishra S, Sabbah HN, Rastogi S, Imai M, Gupta RC (2005) Reduced sarcoplasmic reticulum $\mathrm{Ca} 2+$ uptake and increased $\mathrm{Na}+-\mathrm{Ca} 2+$ exchanger expression in left ventricle myocardium of dogs with progression of heart failure. Heart Vessels 20: 23-32.

Mohr S, Liew CC (2007) The peripheral-blood transcriptome: new insights into disease and risk assessment. Trends Mol Med 13: 422-432.

Moon HS, Choi E, Hyun C (2008) The cardiac sodium-calcium exchanger gene (NCX-1) is a potential canine cardiac biomarker of chronic mitral valvular insufficiency. J Vet Intern Med 22: 1360-1365. 
Nam SJ, Han SH, Kim HW, Hyun C (2010) The cardiac biomarker sodium-calcium exchanger (NCX-1) can differentiate between heart failure and renal failure: a comparative study of NCX-1 expression in dogs with chronic mitral valvular insufficiency and azotemia. J Vet Intern Med 24: 1383-1387.

Obayashi K, Miyagawa-Tomita S, Matsumoto H, Koyama H, Nakanishi T, Hirose H (2011) Effects of transforming growth factor- $\beta 3$ and matrix metalloproteinase- 3 on the pathogenesis of chronic mitral valvular disease in dogs. Am J Vet Res 72: 194-202.

Olsen LH, Fredholm M, Pedersen HD (1999) Epidemiology and inheritance of mitral valve prolapsed in Dachshunds. J Vet Intern Med 13: 448-456.

Oyama MA, Chittur S (2005) Genomic expression patterns of cardiac tissues from dogs with dilated cardiomyopathy. Am J Vet Res 66: 1140-55.

Ritchie ME, Phipson B, Wu D, Hu Y, Law CW, Shi W, Smyth GK (2015) Limma powers differential expression analyses for RNA-sequencing and microarray studies. Nucleic Acids Res 43: e47.
Seiler PU, Stypmann J, Breithardt G, Schulze-Bahr E (2004) Real-time RT-PCR for gene expression profiling in blood of heart failure patients - a pilot study: gene expression in blood of heart failure patients. Basic Res Cardiol 99: $230-238$.

Smyth GK (2004) Linear models and empirical Bayes methods for assessing differential expression in microarray experiments. Stat Appl Genet Mol Biol 3: 1-25.

Stern JA, Hsue W, Song KH, Ontiveros ES, Luis Fuentes V, Stepien RL (2015) Severity of Mitral Valve Degeneration Is Associated with Chromosome 15 Loci in Whippet Dogs. PLoS One; 10: e0141234.

Szmit S, Jank M, Maciejewski H, Balsam P, Majewska A, Łój M, Grabowski M, Filipiak KJ, Motyl T, Opolski G (2012) White blood cell transcriptome correlates with renal function in acute heart failure. Int Heart J 53: 117-124.

Zheng J, Chen Y, Pat B, Dell'italia LA, Tillson M, Dillon AR, Powell PC, Shi K, Shah N, Denney T, Husain A, Dell'Italia LJ (2009) Microarray identifies extensive downregulation of noncollagen extracellular matrix and profibrotic growth factor genes in chronic isolated mitral regurgitation in the dog. Circulation 119: 2086-2095. 